\title{
Avaliação preliminar dos efeitos da ineficiência dos serviços de saneamento na saúde pública brasileira
}

\author{
Patrícia da Silva Figueiredo FERREIRA ${ }^{\prime}$; Patrícia Constantino MOTTA'; Tayane Crispim de SOUZA'; \\ Thiago Paulo da SILVA'; Juliana Ferreira de OLIVEIRA'; Ana Silvia Pereira SANTOS
}

\patriciasff.eng@gmail.com

1. Universidade do Estado do Rio de Janeiro, Rua São Francisco Xavier, 524, Rio de Janeiro, Brasil.

Histórico do Artigo:

Recebido: 26 de julho de 2016

Aceito: 23 de novembro de $2016 \quad$ Publicado: 22 de dezembro de 2016

Resumo: Sendo o saneamento básico um serviço de infraestrutura fundamental na promoção da saúde pública em uma localidade, este trabalho foi realizado com o objetivo de relacionar os impactos na saúde da população e os custos gerados, devido à ineficiência do serviço de saneamento ambiental no Brasil. Destacam-se para a elaboração desse estudo duas etapas: 1) análise do número de casos, por região geográfica, de 10 dentre as principais doenças de veiculação hídrica no período de 2001 a 2010; e 2) comparação entre o número de casos das mesmas doenças com as informações de gastos/investimentos em saúde e saneamento e crescimento populacional no mesmo período. Pode-se verificar que o número de casos ao longo dos anos não sofreu retrocesso significativo, como seria de se esperar. Isso pode ser justificado pela falta de investimentos adequados em saneamento gerando, portanto, uma má prestação dos serviços de coleta e tratamento de esgoto sanitário, de gestão de resíduos sólidos urbanos, de manejo de águas pluviais urbanas e de abastecimento público de água. A ineficiência destes serviços implica diretamente no aumento dos gastos com a saúde pública para tratar os sintomas das doenças de veiculação hídrica, gerando um modelo de gestão insatisfatório. Assim, o estudo afirma a importância do saneamento, como forma preventiva e eficaz, na melhoria dos índices de saúde pública no Brasil.

Palavras-chave: Saneamento, Saúde pública, Esgoto sanitário, Abastecimento de água.

\section{Preliminary evaluate of inefficiency of sanitation services effects in brazilian public health}

\begin{abstract}
Sanitation is a fundamental infrastructure service of public health promotion in a community. This article demonstrates the impacts of the lack of sanitation services on its population's health considering the costs involved in Brazil. This study is divided in two segments: 1) analysis of the number of cases by geographic region of the top 10 most expressive waterborne diseases from 2001 to 2010; and 2) comparison between the number of cases of the same diseases with the spending/investments information on health and sanitation according government sources, considering population growth in same period. The results indicate that the number of cases over the years did not suffer a significant setback as expected. This situation can be explained by the lack of adequate investment in sanitation, expressed by poor services in sewage and solid waste treatments, faulty management of urban rainwater and deficient monitoring and maintenance of public water supply installations. The inefficiency of these services directly affects the increase on health spending from waterborne diseases treatments, consequently being an unsatisfactory management model. Therefore, this study affirms the importance of sanitation as a preventive and effective way on the improvement of public health rates in Brazil.

Keywords: Sanitation, Public health, Sewage, Water supply.
\end{abstract}




\title{
Evaluación preliminar de los efectos de la ineficiencia de los servicios de saneamiento en la salud pública brasileña
}

\begin{abstract}
Resumen: El saneamiento es un servicio de infraestructura fundamental en la promoción de la salud pública en un lugar. En este trabajo se llevó a cabo con el fin de relacionar los impactos sobre la salud de la población y los costos incurridos debido a la ineficiencia de los servicios de saneamiento ambiental en Brasil. Se destacan para la elaboración de este estudio dos etapas: 1) el número de casos, el análisis por cada región geográfica, de 10 de las principales enfermedades transmitidas por el agua en el período 2001 hasta 2010; y 2) comparación entre el número de casos de las respectivas enfermedades con las informaciones de gastos/inversiones en la salud y saneamiento y crecimiento de la población en el mismo período. Se puede observar que el número de casos en los años no sufrió revés significativo, como sería de esperar. Esto puede explicarse por la falta de una adecuada inversión en saneamiento y, por lo tanto, genera una mala prestación de los servicios de colecta y tratamiento de aguas residuales domésticas, la gestión de residuos sólidos, la gestión del agua de lluvia urbana y abastecimiento público de agua. La ineficiencia de estos servicios implica directamente el aumento del gasto en salud pública para tratar los síntomas de las enfermedades transmitidas por el agua, la generación de un modelo de gestión insatisfactoria. Por lo tanto, el estudio confirma la importancia de la higiene como medida preventiva y eficaz, mejorando los índices de salud pública en Brasil.
\end{abstract}

Palabras clave: Saneamiento, Salud pública, Aguas residuales domésticas, Abastecimiento de agua.

\section{INTRODUÇÃ0}

0 termo saneamento básico, sempre foi designado à prestação de serviços à população, tais como abastecimento de água, esgotamento sanitário, gestão de resíduos sólidos e manejo de águas pluviais urbanas, com o intuito de promoção à saúde, segurança à vida, ao patrimônio público e privado, além da proteção ambiental. Em 2007, foi promulgada a Lei 11.445/2007 (BRASIL, 2007), que estabelece diretrizes nacionais para o saneamento básico e apresenta para ele, uma definição mais completa. Neste caso, os serviços públicos de saneamento devem ser prestados com base em princípios tais como: i) Universalização dos sistemas, segundo uma ampliação progressiva do acesso de todos os domicílios ocupados; ii) Integralidade, compreendida como o conjunto de todas as atividades e componentes de cada um dos serviços de saneamento básico; iii) Adoção de métodos, técnicas e processos que considerem as peculiaridades locais e regionais; iv) Articulação com as políticas de desenvolvimento urbano e regional, de habitação, de combate à pobreza, de proteção ambiental e de promoção à saúde; v) Eficiência e sustentabilidade econômica; vi) Utilização de tecnologias apropriadas, considerando a capacidade de pagamento dos usuários; vii) Transparência das ações, segurança, qualidade e regularidade; e viii) Integração das infraestruturas e serviços com a gestão eficiente dos recursos hídricos.

Nos países mais pobres e em desenvolvimento, o assunto é negligenciado ainda nos dias atuais e poucos investimentos públicos são direcionados a esse setor. Haja vista o quadro do 
saneamento ambiental no Brasil que apresenta um cenário insatisfatório. Segundo o Sistema Nacional de Informações sobre Saneamento - SNIS, em seu último Diagnóstico dos Serviços de Água e Esgoto - 2014 (SNIS, 2016), lançado em fevereiro de 2016, 93,2\% da população urbana é atendida por rede de abastecimento de água e somente $57,6 \%$ dessa mesma população é atendida com coleta de esgoto. Já em relação à população total (população urbana + rural) estes índices representam respectivamente 83,0\% e 49,8\%. Essa análise em relação à população total é relevante e ainda mais preocupante, na medida em que é sabido que uma parte significativa da população brasileira vive em áreas rurais. De acordo com o IBGE (2010), até houve uma redução da porcentagem de população rural entre os anos 2000 e 2010, passando de 18,77\% para 15,64\%. Entretanto ainda é uma parcela considerável.

Já em relação ao esgoto gerado, somente 40,8\% sofre algum tipo de tratamento e neste cenário, quase $60 \%$ do esgoto doméstico gerado no Brasil é lançado in natura nos corpos d'água. Por fim, somente 70,9\% de todo esgoto coletado no Brasil recebe algum tipo de tratamento (SNIS, 2016).

Esses dados apresentam somente índices de atendimento. Porém é importante ressaltar a qualidade da prestação desses serviços, principalmente em relação à qualidade da água oferecida para abastecimento doméstico e das características dos efluentes de Estações de Tratamento de Esgotos, lançados em corpos d'água.

A falta de saneamento básico ou a ineficiência da prestação desses serviços, contribui fortemente para a precariedade da saúde pública de uma localidade. Isso fica bastante evidenciado no Brasil ao se observar a qualidade da maioria dos corpos d'água urbanos, a qualidade de vida do cidadão e o elevado nível de susceptibilidade da população às doenças de veiculação hídrica, que segundo Fundação Nacional de Saúde (FUNASA, 2010) são classificadas como Doenças Relacionadas ao Saneamento Ambiental Inadequado, ou simplesmente DRSAI.

Grande parte das doenças registradas pela falta de saneamento é de transmissão orofecal e apresenta como sintoma, além de outros, a diarreia, que é bastante característico desse grupo de enfermidades. Mais recentemente, doenças como a Dengue e a Zika se transformaram em grandes epidemias nacionais, trazendo graves consequências para a população brasileira. Essas também tornaram a falta de saneamento, que era algo praticamente invisível, em algo visível e mensurável, através de outros sintomas como a febre, as dores nas articulações, as erupções na pele, dores de cabeça e óbitos registrados no Sistema de Saúde. 
Avaliação preliminar dos efeitos da ineficiência dos serviços de saneamento na saúde pública brasileira

Assim, essas doenças são classificadas em geral, como de transmissão oro-fecal e/ou por inseto-vetor e são relacionadas à falta de higiene que em sua maioria são passíveis de erradicação através de um meio saneado.

As DRSAI são notificadas em países considerados pobres e subdesenvolvidos, com serviços de saneamento precários, falta de políticas públicas e falta de educação sanitária da população. Esses fatores favorecem um cenário propício ao desenvolvimento dessas doenças e ao agravamento do quadro desfavorável em relação à saúde pública no país.

São diversas as doenças relacionadas ao saneamento inadequado e algumas classificações já foram realizadas por outros autores. Cairncross e Feachem (1993) realizaram a classificação ambiental considerada a mais importante para doenças infecciosas, que se baseia em enfermidades relacionadas à água, excreta e lixo. Dessa forma, Heller (1997) indica que diferente da classificação biológica clássica, por agentes etiológicos, a delimitação proposta pelos autores toma por base as vias de transmissão, o ciclo biológico e as principais estratégias de controle ambiental dessas doenças. Dessa forma, a classificação proposta por Cairncross e Feachem (1993), pode ser observada em três principais classes:

1) Classe de doenças relacionadas à água com quatro categorias de transmissão: i) orofecal determinada pela ingestão ou contato com a água; ii) relacionadas com a higiene, onde a infecção acontece principalmente por pele e olhos; iii) baseada na água por penetração na pele ou por ingestão; iv) através de inseto vetor por picadura, próxima à água ou que procriam na água. Essas doenças são passíveis de controle, ou pelo menos podem ser influenciadas através das águas de abastecimento.

2) Classe de doenças relacionadas às excretas que reúne aquelas causadas por patógenos transmitidos por excretas humanas, normalmente as fezes, e possibilita a compreensão de medidas sanitárias relacionadas com a eliminação de excretas.

3) Classe de doenças transmitidas por insetos vetores e roedores estão relacionadas à moradia, à água e às excretas, cuja transmissão ocorre por roedores em locais onde vetores contaminados por fezes podem se procriar. A melhoria do acondicionamento e da coleta de lixo, bem como o controle de roedores são medidas de controle dessas doenças. 
Na Tabela 1 podem ser observadas as doenças, que ao longo do presente trabalho serão analisadas em relação ao número de casos entre os anos de 2001 e 2010, frente principalmente aos investimentos em saneamento e saúde realizados no mesmo período.

Segundo Escola Nacional de Saúde Pública (ENSP, 2013) a cada 15 segundos uma criança morre no mundo por doenças relacionadas à falta de água potável, saneamento e condições de higiene.

0 levantamento da ENSP (2013) aponta que no mundo, cerca de 900 milhões de pessoas não têm acesso à água potável, o que equivale à quase cinco vezes a população brasileira, enquanto que 125 milhões de crianças menores de cinco anos vivem em lares sem acesso a fontes de água potável. Ainda, mais de 2,5 bilhões de pessoas não possuem serviços dignos de saneamento, o que equivale a 1/3 da população mundial.

Dessa forma, o presente estudo tem o objetivo de avaliar os efeitos da prestação inadequada dos serviços de saneamento na saúde pública brasileira, a partir das relações entre três aspectos: i) número de casos de DRSAI, ii) investimentos em saneamento e saúde e iii) crescimento populacional no período compreendido entre 2001 e 2010.

Tabela 1 - Relação das doenças que serão analisadas ao longo do presente trabalho e suas principais características

\begin{tabular}{|c|c|c|c|}
\hline DOENÇA & CATEG0RIA* & FORMA DE TRANSMISSÃO & AGENTE \\
\hline Dengue & $\begin{array}{l}\text { Inseto vetor } \\
\text { procria na água }\end{array}$ & $\begin{array}{l}\text { A transmissão do hospedeiro vertebrado } \\
\text { para o vetor ocorre quando a fêmea se } \\
\text { contamina ao picar um indivíduo } \\
\text { infectado tornando-se capaz de } \\
\text { transmitir o vírus por meio da saliva } \\
\text { contaminada. }\end{array}$ & $\begin{array}{l}\text { Vírus } \\
\text { Dengue }\end{array}$ \\
\hline Doença de Chagas & $\begin{array}{l}\text { Inseto vetor } \\
\text { procria na água }\end{array}$ & $\begin{array}{l}\text { Geralmente transmitida pelo barbeiro. } \\
\text { Contudo, em alguns casos raros, a } \\
\text { transmissão da moléstia pode ser } \\
\text { congênita, por transfusão de sangue, } \\
\text { transplante de órgãos, acidentes de } \\
\text { laboratórios ou com a ingestão de } \\
\text { alimentos contaminados com insetos } \\
\text { infectados. }\end{array}$ & $\begin{array}{l}\text { Protozoário } \\
\text { Trypanosoma } \\
\text { cruzi }\end{array}$ \\
\hline Esquistossomose & $\begin{array}{l}\text { Baseada na água } \\
\text { por penetração na } \\
\text { pele ou ingestão }\end{array}$ & $\begin{array}{l}0 \text { agente etiológico penetra pela pele ou é } \\
\text { ingerido. }\end{array}$ & $\begin{array}{l}\text { Helminto } \\
\text { Schistosoma } \\
\text { mansoni }\end{array}$ \\
\hline Febre Amarela & $\begin{array}{l}\text { Inseto vetor } \\
\text { procria na água }\end{array}$ & $\begin{array}{l}\text { Transmitida ao homem pela picada de } \\
\text { fêmeas de mosquitos do gênero } \\
\text { Haemagogus nas Américas e Aedes, na }\end{array}$ & $\begin{array}{l}\text { Vírus } \\
\text { Amarílico }\end{array}$ \\
\hline
\end{tabular}




\begin{tabular}{|c|c|c|c|}
\hline & & $\begin{array}{l}\text { África, infectadas ao sugar primatas, que } \\
\text { são os hospedeiros bem como } \\
\text { amplificadores e disseminadores do vírus } \\
\text { nas florestas. }\end{array}$ & \\
\hline Febre Tifóide & Oro-fecal & $\begin{array}{l}0 \text { contágio se dá por meio fecal-oral em } \\
\text { relação à água contaminada } \operatorname{com~a~} \\
\text { bactéria }\end{array}$ & $\begin{array}{l}\text { Bactéria } \\
\text { Salmonella typhi }\end{array}$ \\
\hline Hepatite & Oro-fecal & $\begin{array}{l}\text { Ingestão com contaminantes, má higiene } \\
\text { dos alimentos e a forma de tratamento } \\
\text { dos dejetos. }\end{array}$ & $\begin{array}{l}\text { Vírus } \\
\mathrm{HAV}, \mathrm{HBV} \text { e HCV }\end{array}$ \\
\hline $\begin{array}{l}\text { Leishmaniose } \\
\text { Visceral e } \\
\text { Tegumentar }\end{array}$ & $\begin{array}{l}\text { Inseto vetor } \\
\text { procria na água }\end{array}$ & $\begin{array}{l}\text { Transmitidas por insetos hematófagos. As } \\
\text { fontes de infecção são, principalmente, os } \\
\text { animais silvestres e os insetos } \\
\text { flebotomíneos, porém, o hospedeiro } \\
\text { também pode ser o cão doméstico. } 0 \\
\text { inseto que abriga o parasita pica o } \\
\text { hospedeiro final, transmitindo a doença. }\end{array}$ & $\begin{array}{l}\text { Protozoário do } \\
\text { gênenor } \\
\text { Leishmania }\end{array}$ \\
\hline Leptospirose & $\begin{array}{l}\text { Oro-fecal ou } \\
\text { vetores/roedores }\end{array}$ & $\begin{array}{l}0 \text { agente etiológico penetra pela pele ou é } \\
\text { ingerido. }\end{array}$ & $\begin{array}{l}\text { Bactéria } \\
\text { gênero } \\
\text { Leptospira }\end{array}$ \\
\hline Malária & $\begin{array}{l}\text { Inseto vetor } \\
\text { próximo a água }\end{array}$ & $\begin{array}{l}\text { Transmissão por meio da picada da fêmea } \\
\text { do mosquito Anopheles, infectada pelo } \\
\text { Plasmodium. }\end{array}$ & $\begin{array}{l}\text { Protozoário } \\
\text { Plasmodium } \\
\text { malariae }\end{array}$ \\
\hline
\end{tabular}

Fonte: HELLER e CASSEB, 2001.

Observação: *Classificação de acordo com Cairncross e Feachem (1993).

\section{MATERIAL E MÉTODOS}

0 trabalho foi desenvolvido a partir de uma análise do banco de dados do Departamento de Informática do Sistema Único de Saúde - DATASUS com informações sobre as doenças registradas no Brasil. Para o caso deste trabalho, foram analisadas somente algumas das principais doenças de veiculação hídrica em um horizonte de 10 anos, compreendido entre 2001 e 2010.

Tanto o período de análise quanto a seleção das doenças foram adotados em função do banco de dados do DATASUS apresentar informações mais completas em relação ao número de casos registrados no país para cada uma das doenças abordadas na pesquisa. São elas: Dengue, Doença de Chagas, Esquistossomose, Febre Amarela, Febre Tifóide, Hepatite, Leishmaniose Visceral, Leishmaniose Tegumentar, Leptospirose e Malária.

Para uma análise completa das implicações da falta de investimentos no setor saneamento, os dados sobre as doenças de veiculação hídrica registradas no país no período já mencionado foram confrontados com dados relacionados aos gastos/investimentos federais em 
saúde e em saneamento no mesmo período. Estes dados foram extraídos da Rede Interagencial de Informações para a Saúde - RIPSA (2012).

0 presente trabalho foi então desenvolvido em duas etapas como descritas:

Etapa 01 - Análise do número de casos de cada uma das doenças estudadas, por região geográfica, e no período compreendido entre 2001 a 2010.

Etapa 02 - Comparação entre o número de casos das doenças analisadas no estudo, no período de 2001 a 2010, com as informações de gastos/investimentos em saúde e saneamento, e crescimento populacional no mesmo período.

\section{RESULTADOS E DISCUSSÃO}

Etapa 01 - Análise do número de casos de cada uma das doenças estudadas, por região geográfica, e no período compreendido entre 2001 a 2010.

Na Tabela 2 está apresentado o número de casos de cada uma das doenças estudadas, ano a ano, em todas as regiões geográficas do Brasil.

Tabela 2 - Número de casos de doenças de veiculação hídrica nas regiões geográficas do Brasil, de 2001 a 2010

\begin{tabular}{|c|c|c|c|c|c|c|c|c|c|c|c|}
\hline \multirow{2}{*}{ ANO } & \multirow{2}{*}{ REGIÃO } & \multicolumn{10}{|c|}{ DOENÇA } \\
\hline & & $\mathrm{D}$ & $\mathrm{DC}$ & $E$ & $\mathrm{FA}$ & FT & $\mathrm{H}$ & $\mathrm{LV}$ & LT & $\mathrm{L}$ & M \\
\hline \multirow{5}{*}{2001} & $\mathrm{~N}$ & 51859 & 5 & 311 & 9 & 317 & 5131 & 352 & 9500 & 142 & - \\
\hline & $\mathrm{NE}$ & 149752 & 37 & 29837 & - & 43 & 9492 & 2101 & 14387 & 662 & 20 \\
\hline & SE & 159715 & 5 & 15601 & 26 & 12 & 10445 & 270 & 2760 & 1178 & 104 \\
\hline & S & 1690 & 12 & 189 & $\begin{array}{lll}- & \\
\end{array}$ & 8 & 14154 & 3 & 660 & 1654 & 75 \\
\hline & $\mathrm{CO}$ & 23844 & - & 60 & - & - & 4314 & 134 & 5149 & 43 & 63 \\
\hline \multirow{5}{*}{2002} & $\mathrm{~N}$ & 20290 & 38 & 227 & 4 & 497 & 5609 & 431 & 11346 & 224 & - \\
\hline & $\mathrm{NE}$ & 267746 & 114 & 44346 & - & 315 & 9834 & 1671 & 10726 & 639 & 36 \\
\hline & SE & 360109 & 36 & 11435 & 4 & 26 & 7819 & 463 & 3242 & 985 & 389 \\
\hline & S & 8011 & 48 & 142 & - & 18 & 9289 & - & 1049 & 859 & 225 \\
\hline & $\mathrm{CO}$ & 47042 & 10 & 157 & - & 7 & 3330 & 209 & 5285 & 41 & 177 \\
\hline \multirow{5}{*}{2003} & $\mathrm{~N}$ & 28662 & 49 & 257 & 2 & 413 & 5329 & 483 & 14287 & 250 & - \\
\hline & $\mathrm{NE}$ & 151601 & 436 & 42080 & - & 417 & 10144 & 2053 & 8450 & 509 & 167 \\
\hline & $\begin{array}{l}\text { SE } \\
\text {. }\end{array}$ & 66498 & 33 & 10283 & 49 & 21 & 12586 & 572 & 3544 & 1010 & 470 \\
\hline & $\mathrm{S}$ & 11203 & 46 & 378 & - & 15 & 9470 & 2 & 989 & 1231 & 198 \\
\hline & $\mathrm{CO}$ & 21175 & 15 & 49 & 4 & 3 & 3913 & 234 & 5338 & 50 & 241 \\
\hline \multirow{3}{*}{2004} & $\mathrm{~N}$ & 19230 & 38 & 335 & 3 & 322 & 7750 & 576 & 13719 & 226 & - \\
\hline & $\mathrm{NE}$ & 22364 & 264 & 38799 & - & 215 & 12356 & 2044 & 8230 & 805 & 299 \\
\hline & SE & 21664 & 42 & $\begin{array}{l}9400 \\
\end{array}$ & 1 & 21 & 18414 & 794 & 2751 & 1315 & 631 \\
\hline
\end{tabular}


Avaliação preliminar dos efeitos da ineficiência dos serviços de saneamento na saúde pública brasileira

\begin{tabular}{|c|c|c|c|c|c|c|c|c|c|c|c|}
\hline & $\mathrm{S}$ & 198 & 58 & 251 & - & 10 & 11523 & 5 & 647 & 672 & 245 \\
\hline & $\mathrm{CO}$ & 8870 & 17 & 123 & - & 3 & 4592 & 357 & 4793 & 77 & 314 \\
\hline \multirow{5}{*}{2005} & $\mathrm{~N}$ & 25966 & 52 & 219 & 4 & 203 & 7210 & 693 & 11247 & 247 & - \\
\hline & $\mathrm{NE}$ & 76071 & 426 & 38418 & - & 249 & 15699 & 2102 & 8550 & 745 & 244 \\
\hline & SE & 21353 & 57 & 8167 & 1 & 37 & 20569 & 671 & 3045 & 1336 & 833 \\
\hline & $\mathrm{S}$ & 1157 & 78 & 367 & - & 5 & 13760 & 3 & 594 & 1072 & 284 \\
\hline & $\mathrm{CO}$ & 26499 & 16 & 129 & - & - & 7086 & 381 & 4664 & 64 & 325 \\
\hline \multirow{5}{*}{2006} & $\mathrm{~N}$ & 20776 & 132 & 115 & 1 & 145 & 7942 & 758 & 9264 & 781 & - \\
\hline & $\mathrm{NE}$ & 69703 & 299 & 43755 & - & 422 & 11379 & 2063 & 6550 & 693 & 231 \\
\hline & $\mathrm{SE}$ & 128394 & 44 & 5460 & - & 23 & 21345 & 730 & 3041 & 1773 & 751 \\
\hline & $\mathrm{S}$ & 1373 & 45 & 349 & - & 10 & 11855 & 3 & 604 & 1217 & 232 \\
\hline & $\mathrm{CO}$ & 46127 & 24 & 88 & 1 & 1 & 4778 & 372 & 4124 & 75 & 237 \\
\hline \multirow{5}{*}{2007} & $\mathrm{~N}$ & 35428 & 151 & 62 & 3 & 111 & 3898 & 801 & 10302 & 243 & - \\
\hline & $\mathrm{NE}$ & 118458 & 3 & 26603 & - & 251 & 5755 & 1726 & 6272 & 537 & 178 \\
\hline & $\mathrm{SE}$ & 199088 & - & 6155 & - & 31 & 14568 & 690 & 2112 & 1233 & 545 \\
\hline & $\mathrm{S}$ & 27567 & - & 314 & - & 9 & 8371 & 4 & 557 & 1241 & 250 \\
\hline & $\mathrm{CO}$ & 101349 & 1 & 69 & 2 & 2 & 2897 & 341 & 3313 & 38 & 200 \\
\hline \multirow{5}{*}{2008} & $\mathrm{~N}$ & 46598 & 86 & 80 & 2 & 97 & 4273 & 861 & 9270 & 311 & - \\
\hline & $\mathrm{NE}$ & 193992 & 8 & 5335 & - & 208 & 6241 & 1893 & 6605 & 587 & 136 \\
\hline & SE & 297187 & - & 6928 & 12 & 34 & 15090 & 836 & 1791 & 997 & 458 \\
\hline & S & 2019 & - & 172 & 5 & 5 & 8730 & 4 & 670 & 1547 & 119 \\
\hline & $\mathrm{CO}$ & 45982 & - & 37 & 27 & 2 & 2923 & 396 & 3245 & 50 & 126 \\
\hline \multirow{5}{*}{2009} & $\mathrm{~N}$ & 52968 & 201 & 96 & - & 118 & 4924 & 774 & 8842 & 354 & - \\
\hline & $\mathrm{NE}$ & 118658 & 4 & 2512 & - & 152 & 6434 & 1939 & 7384 & 918 & 182 \\
\hline & SE & 105981 & - & 15149 & 30 & 23 & 16584 & 824 & 1811 & 1497 & 403 \\
\hline & $\mathrm{S}$ & 1589 & - & 269 & 18 & 6 & 9201 & 10 & 521 & 1088 & 146 \\
\hline & $\mathrm{CO}$ & 106953 & 1 & 44 & 2 & 5 & 3701 & 345 & 4760 & 49 & 130 \\
\hline \multirow{5}{*}{2010} & $\mathrm{~N}$ & 97675 & 176 & 60 & 1 & 65 & 4034 & 701 & 7632 & 232 & - \\
\hline & $\mathrm{NE}$ & 169598 & 12 & 1488 & - & 72 & 4517 & 1845 & 9453 & 670 & 300 \\
\hline & SE & 169598 & - & 22564 & - & 9 & 14634 & 810 & 2654 & 1385 & 545 \\
\hline & $\mathrm{S}$ & 41639 & - & 69 & 1 & 5 & 9818 & 7 & 304 & 1125 & 182 \\
\hline & $\mathrm{CO}$ & 215770 & 7 & 33 & 1 & 3 & 2338 & 338 & 3450 & 44 & 205 \\
\hline
\end{tabular}

Fonte: DATASUS, 2016.

Observações: Doenças: D - Dengue, DC - Doença de Chagas, E - Esquistossomose, FA - Febre Amarela, FT - Febre Tifóide, H - Hepatite, LV - Leishmaniose Visceral, LT - Leishmaniose Tegumentar, L - Leptospirose, M - Malária. Regiões Geográficas: N - Norte, NE - Nordeste, SE, Sudeste, S - Sul, C0 - Centro Oeste

Já o gráfico da Figura 1 representa o somatório do número de casos de todas as doenças estudadas, por ano (no período de estudo) em cada uma das regiões geográficas brasileiras.

É possível destacar que, tanto na região nordeste como na região sudeste, o maior número de casos dessas doenças aconteceu no ano de 2002, seguido pelo ano de 2008. Nas outras regiões (norte, sul e centro-oeste) o maior número de casos registrados ocorreu em 2010. Pode-se observar que tanto em 2002 como em 2008, os resultados apresentados para as regiões nordeste e sudeste sofreram forte influência da Dengue que neste período apresentou número 
de casos bastante elevados. Ressalta-se ainda que, em relação a todo o período estudado, desde 2001 até 2010, os casos de dengue no Brasil representam 77\% de todas as doenças analisadas no presente estudo.

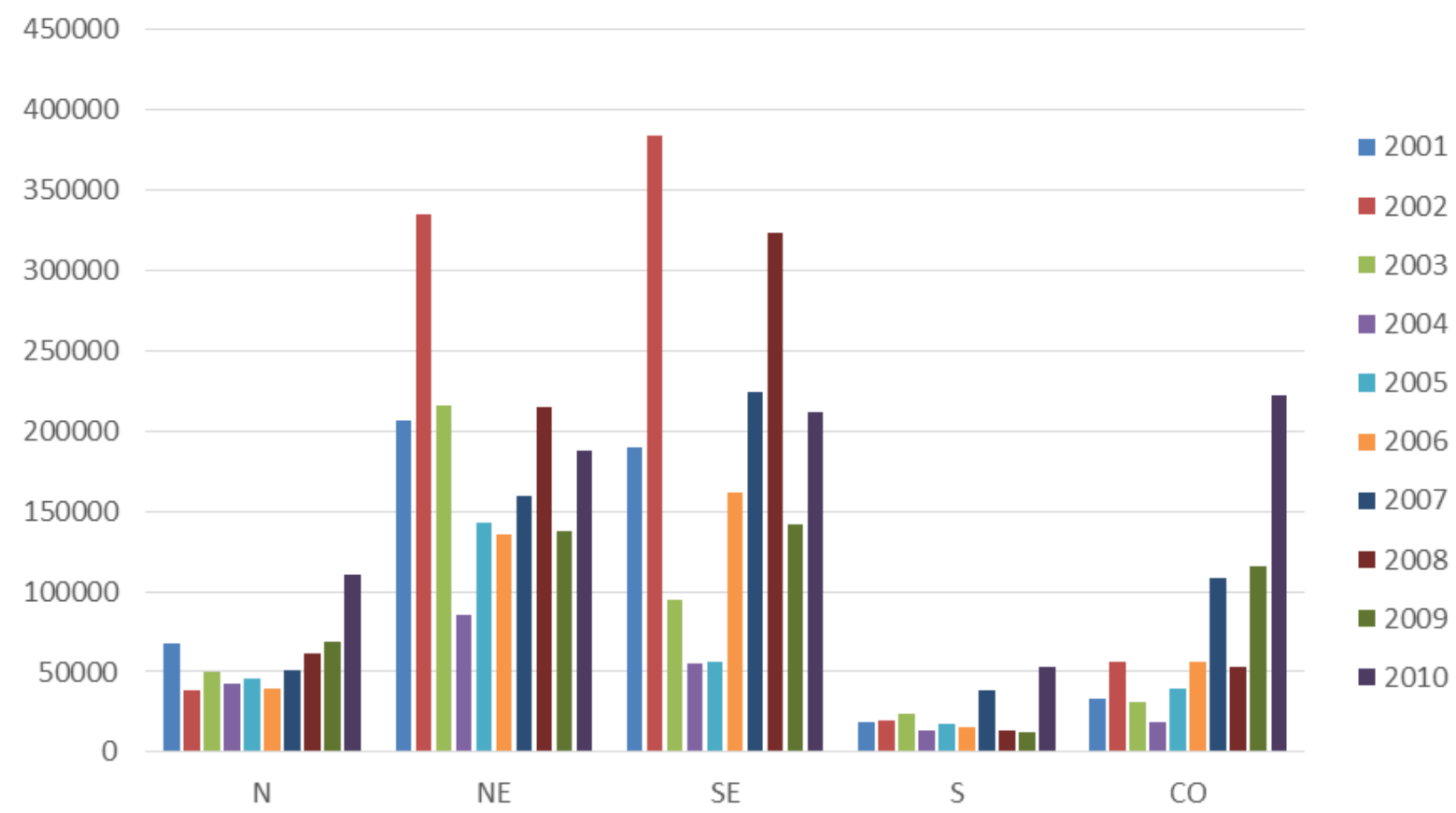

Figura 1. Gráfico do somatório do número de casos das doenças estudadas nas regiões geográficas do Brasil no período de 2001 a 2010

Fonte: DATASUS, 2016.

Uma análise mais criteriosa para comparação entre as regiões deve ser realizada em função do número de casos totais registrados em relação à população total da região. Essa análise foi realizada somente para o ano de 2010 e dessa forma, foi elaborada a Tabela 3, que apresenta um resumo do número total de casos das doenças estudadas registradas em 2010 e a população da região no mesmo ano. Para facilitar o entendimento, foi ainda apresentada uma relação entre o número de casos total de doenças naquele ano e a população total da região.

Foi possível observar que para o ano de 2010 a região centro-oeste apresentou o maior número de casos (222.189), e quando analisado em relação à população total da região, este índice, de 1,58\% também foi o maior dentre todas as regiões. Porém ao se observar a região norte, percebe-se que apesar de apresentar um dos números totais de casos mais baixos dentre as demais regiões, superando somente a região sul, apresenta índice elevado quando se compara o número total de casos à população total da região. Este índice foi de $0,70 \%$, superando as regiões 
Avaliação preliminar dos efeitos da ineficiência dos serviços de saneamento na saúde pública brasileira

sudeste e nordeste que respectivamente apresentaram índices de 0,35\% e 0,26\%. Sobre a região sul, é possível afirmar que além de apresentar o menor número de casos em 2010 (53.150), também apresentou o menor índice em relação à população total, de 0,19\%.

Tabela 3 - Relação entre número de casos de todas as doenças estudadas e a população total de cada região em 2010

\begin{tabular}{|c|c|c|c|c|c|}
\hline & & & POPULACૂÃO & $\mathrm{N}^{\circ} \mathrm{CASOS}(2010)$ & $\mathrm{N}^{\circ} \mathrm{CASOS} / \mathrm{POP}$ \\
\hline REGIÃO & ESTAD0 & POPULAÇÃO & DA REGIÃ0 & N CABOS (ZVIO) & TH CADOAT TI \\
\hline \multirow[t]{7}{*}{ Norte } & Amazonas & 3.483 .985 & \multirow{7}{*}{15.864 .454} & \multirow{7}{*}{110.576} & \multirow{7}{*}{$0,70 \%$} \\
\hline & Acre & 733.559 & & & \\
\hline & Rondônia & 1.562 .409 & & & \\
\hline & Roraima & 450.479 & & & \\
\hline & Tocantins & 1.383 .445 & & & \\
\hline & Amapá & 669.526 & & & \\
\hline & Pará & 7.581.051 & & & \\
\hline \multirow[t]{9}{*}{ Nordeste } & Maranhão & 6.574 .789 & \multirow{9}{*}{53.081 .950} & \multirow{9}{*}{187.955} & \multirow{9}{*}{$0,35 \%$} \\
\hline & Piauí & 3.118 .360 & & & \\
\hline & Ceará & 8.452 .381 & & & \\
\hline & R. G. do Norte & 3.168 .027 & & & \\
\hline & Paraíba & 3.766 .528 & & & \\
\hline & Pernambuco & 8.796 .448 & & & \\
\hline & Alagoas & 3.120 .494 & & & \\
\hline & Sergipe & 2.068 .017 & & & \\
\hline & Bahia & 14.016 .906 & & & \\
\hline \multirow[t]{4}{*}{ Sudeste } & Minas Gerais & 19.597 .330 & \multirow{4}{*}{80.364 .410} & \multirow{4}{*}{212.199} & \multirow{4}{*}{$0,26 \%$} \\
\hline & Espírito Santo & 3.514 .952 & & & \\
\hline & Rio de Janeiro & 15.989.929 & & & \\
\hline & São Paulo & 41.262.199 & & & \\
\hline \multirow[t]{3}{*}{ Sul } & Paraná & 10.444 .526 & \multirow{3}{*}{27.386 .891} & \multirow{3}{*}{53.150} & \multirow{3}{*}{$0,19 \%$} \\
\hline & Santa Catarina & 6.248 .436 & & & \\
\hline & R. Grande do Sul & 10.693 .929 & & & \\
\hline \multirow[t]{4}{*}{ Centro 0este } & M. Grosso do Sul & 2.449 .024 & \multirow{4}{*}{14.058 .094} & \multirow{4}{*}{222.189} & \multirow{4}{*}{$1,58 \%$} \\
\hline & M. Grosso & 3.035 .122 & & & \\
\hline & Goiás & 6.003 .788 & & & \\
\hline & Distrito Federal & 2.570 .160 & & & \\
\hline
\end{tabular}

Fonte: DATASUS, 2016; IBGE, 2010.

Percebe-se também, claramente, que em todos os anos do período estudado, a região sul apresentou o menor número de casos dessas doenças, assim como em 2010. Já a região nordeste apresentou o maior número de casos, no período entre 2001 e 2006 e, a partir do ano 2007, esse número foi superado pela região sudeste. 
Pode-se observar também que não há uma tendência de elevação ou de redução do número de casos das doenças estudadas, de uma maneira geral, ao longo do período analisado. Ao longo do desenvolvimento socioeconômico de uma nação, espera-se uma redução do número de casos dessas doenças ao longo dos anos, em função de investimentos na área de saneamento e saúde. No entanto, no gráfico da Figura 2, pode-se observar que entre os anos de 2004 e 2010, houve na verdade, uma elevação do número de casos dessas doenças no Brasil, exceto no ano de 2009.

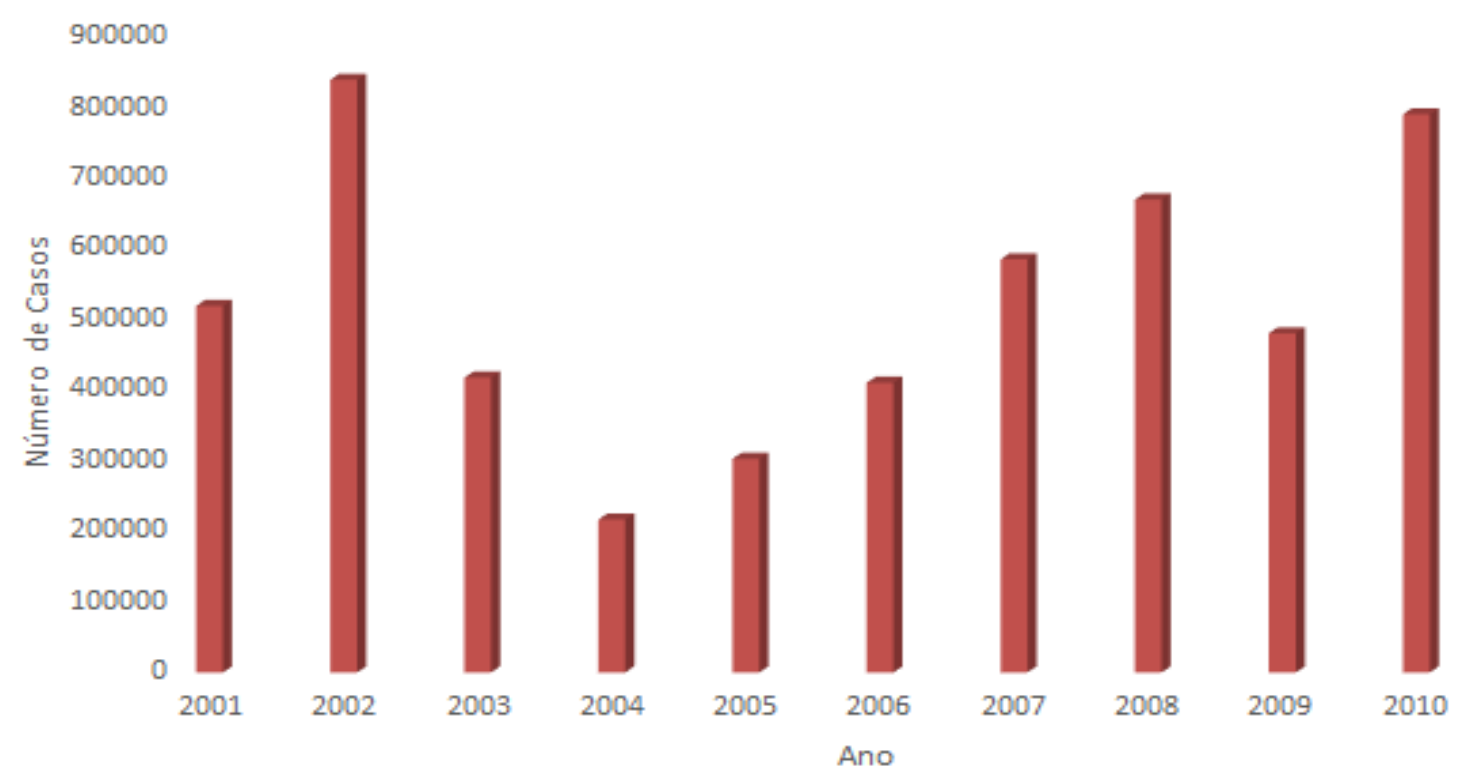

Figura 2. Gráfico do número de casos no Brasil de todas as doenças analisadas, no período de 2001 a 2010

Fonte: DATASUS, 2016.

Etapa 02 - Comparação entre o número de casos das doenças analisadas no estudo, no período de 2001 a 2010, com as informações de gastos/investimentos em saúde e saneamento, e crescimento populacional no mesmo período.

Na Tabela 4 estão apresentados os valores investidos em saúde e em saneamento nos anos de 2001 a 2010, de acordo com RIPSA - Rede Interagencial de Informações para a Saúde (2012), além da projeção populacional para o mesmo período, segundo o IBGE. 0 gráfico da Figura 3 apresenta a comparação entre a projeção populacional e os investimentos realizados em saúde e em saneamento. Ressalta-se que a análise foi realizada em relação à projeção populacional e não em relação à população contada, visto que nesses anos não houve contagem pelo IBGE. 
Avaliação preliminar dos efeitos da ineficiência dos serviços de saneamento na saúde pública brasileira

Tabela 4 - Investimentos realizados no Brasil em saúde e em saneamento no período entre 2001 e 2010 e projeção populacional para o mesmo período

\begin{tabular}{cccc}
\hline \multirow{2}{*}{ ANO } & $\begin{array}{c}\text { INVESTIMENTOS EM } \\
\text { SAÚDE (MILHÕES RS) }\end{array}$ & $\begin{array}{c}\text { INVESTIMENTOS EM } \\
\text { SANEAMENTO (MILHÕES RS) }\end{array}$ & $\begin{array}{c}\text { PROJEÇ̃O } \\
\text { POPULACIONAL }\end{array}$ \\
\hline 2001 & 22.218 & 2.947 & 173.808 .010 \\
\hline 2002 & 24.888 & 1.308 & 176.303 .919 \\
\hline 2003 & 26.870 & 580 & 178.741 .412 \\
\hline 2004 & 31.477 & 1.064 & 181.105 .601 \\
\hline 2005 & 34.101 & 1.705 & 183.383 .216 \\
\hline 2006 & 39.774 & 2.225 & 185.564 .212 \\
\hline 2007 & 44.142 & 4.762 & 187.641 .714 \\
\hline 2008 & 49.531 & 4.964 & 191.480 .630 \\
\hline 2009 & 59.073 & 6.530 & 193.252 .604 \\
\hline 2010 & 63.224 & 4.994 & \\
\hline
\end{tabular}

Fonte: RIPSA, 2012a; RIPSA, 2012b; IBGE, 2008.

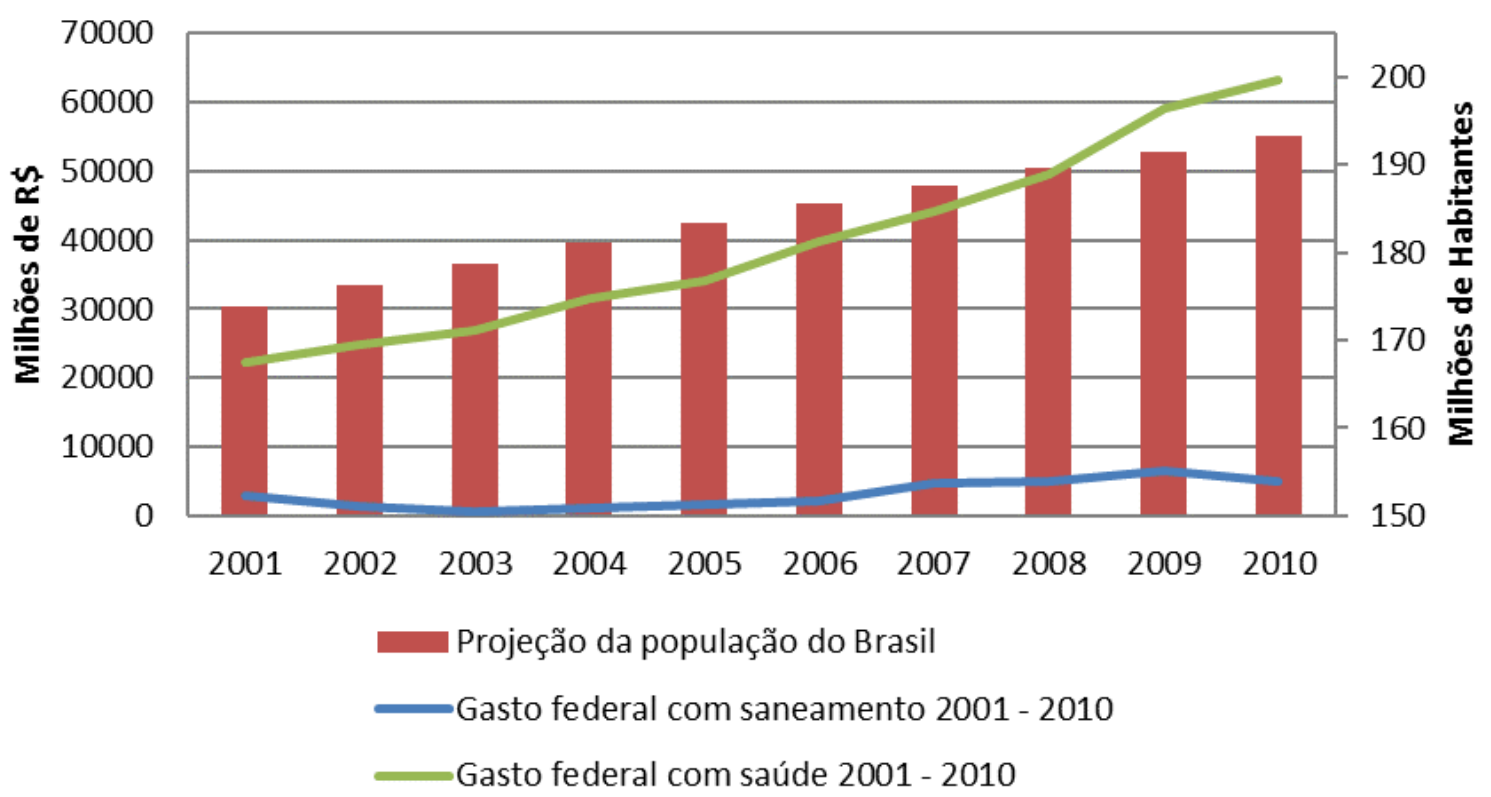

Figura 3. Gráfico da evolução dos investimentos em saúde e saneamento no Brasil entre 2001 e 2010 e a projeção populacional no mesmo período

Fonte: RIPSA, 2012a; RIPSA, 2012b; IBGE, 2008. 
Pode-se perceber uma evolução gradual nos investimentos em saúde, ao longo dos anos, que acontece principalmente em função do crescimento populacional, exceto nos anos de 2009 e 2010. Observa-se nesses dois anos que a taxa de aumento dos investimentos em saúde foi até mais elevado do que a taxa de crescimento populacional.

Já no caso dos investimentos em saneamento uma evolução gradual não é observada. De 2001 a 2010, os investimentos em saúde aumentaram em quase 300\% e, no caso dos investimentos em saneamento, considerando o valor mais baixo investido em 2003 e o mais alto em 2009, houve um aumento acima de 10 vezes. Isso deveria ser considerado interessante, porém ao se analisar esses dados por outro ângulo, percebe-se que ao longo dos anos de 2001 a 2010, o maior investimento em saneamento ocorreu em 2009 no valor de R\$ 6,5 bilhões (já considerado baixo para suprir as necessidades do setor) e o menor investimento ocorreu no ano de 2003, no valor pífio de $R \$ 580$ milhões, 10 vezes inferior.

Somente a título de informação, no Plano Nacional de Saneamento Básico, publicado em 2013 pelo Ministério das Cidades (PLANSAB, 2013), a meta de universalização dos sistemas somente seria alcançada em $2033 \mathrm{com}$ um investimento total em torno de R $\$ 250$ bilhões. Esse montante representa um investimento anual até a data final da ordem de $\mathrm{R} \$ 12,5$ bilhões. Entretanto, ressalta-se que ao longo do período estudado, esses investimentos alcançaram um valor máximo no ano de 2009 abaixo de 7 bilhões e em média, esse investimento anual esteve em torno de 3,1 bilhões.

Destaca-se que ao longo do período estudado, pode-se estimar que foram gastos com saneamento em torno de $\mathrm{R} \$ 17,00$ / habitante e, em relação à saúde, os gastos foram da ordem de $\mathrm{R} \$ 215,00$ / habitante.

Por fim, ao se analisar os gastos federais com saúde no Brasil, há que se ter em mente, que ainda deveria ser computado um gasto com o tratamento arcado pelo próprio indivíduo portador da doença, que mesmo recebendo atenção do Sistema Único de Saúde - SUS ainda deve arcar com remédios, transporte aos hospitais ou postos de saúde, além de equipamentos ou exames não cobertos pelo SUS. Esse é somente o desgaste financeiro do indivíduo. Não se deve esquecer o desgaste emocional e de bem estar tanto do paciente como dos familiares em um tratamento de uma doença de veiculação hídrica que em muitos casos poderia ser evitada simplesmente com uma prestação de serviços de saneamento adequada. 


\section{CONCLUSÃ0}

0 estudo apresentou uma análise da prestação inadequada dos serviços de saneamento no Brasil e sua consequência em relação à saúde da população. Foi apresentado o número de casos de um grupo de doenças de veiculação hídrica registradas no Brasil entre 2001 e 2010, além dos investimentos realizados no país no mesmo período, em saúde e saneamento.

Pôde-se perceber que o número de doenças, dentre aquelas analisadas no presente estudo, não sofreu uma tendência de elevação ou redução ao longo dos anos, apesar de se esperar uma redução quando se trata de um país que vem se desenvolvendo economicamente no cenário mundial.

As regiões nordeste e sudeste do Brasil, por serem as mais populosas, apresentam 0 maior número de casos de doenças em praticamente todos os anos do período de estudo. Porém no ano de 2010, esse número foi superado pela região centro-oeste. Pode-se perceber que essa ocorrência na região centro-oeste se deu principalmente em relação ao aumento significativo do número de casos de dengue que de 2009 para 2010 praticamente dobrou.

Quando se compara o número de casos com a população da região, como realizado para o ano de 2010, novamente a região centro-oeste, mesmo apresentando a menor concentração populacional, foi mais vulnerável, seguida da região norte, do nordeste, do sudeste e por fim da região sul.

Ainda, em relação à região sul, observa-se o menor número de casos de doenças quando comparada às demais regiões, em todos os anos do período de estudo. E mesmo apresentando a terceira maior concentração populacional em relação às 5 regiões, ao se comparar o número de casos com a população da região, esta também apresenta o menor índice dentre todas as regiões geográficas brasileiras. Destaca-se que conhecidamente a região sul é a mais desenvolvida do país com índices de educação e escolaridade mais elevados e nestes casos de prevenção das doenças de veiculação hídrica, o nível educacional é fundamental.

Por fim, é sabido que o investimento adequado em saneamento ambiental em uma determinada região proporciona mais saúde à sua população. No entanto, no Brasil, pode-se observar que os investimentos em saneamento ainda são muito aquém do desejado para se 
alcançar a universalização dos sistemas. Conforme mencionado, o PLANSAB definiu um investimento mínimo da ordem de $R \$ 12,5$ bilhões por ano e entre 2001 e 2010 o que se viu foi um investimento anual médio da ordem de R\$ 3,1 bilhões.

Em contrapartida, conforme apresentado no trabalho, a média brasileira de gastos por habitante com saúde, no período estudado, foi da ordem de $\mathrm{R} \$ 215,00$ / habitante. Por se tratar de investimentos em saúde como um todo e não especificamente em saúde pública, este valor é demasiadamente elevado. Porém de qualquer maneira poderia ser reduzido caso houvesse uma maior preocupação com o saneamento ambiental no Brasil.

\section{REFERÊNCIAS BIBLIOGRÁFICAS}

BRASIL. Lei $\mathrm{n}^{0} 11.445$ de 5 de janeiro de 2007. Estabelece diretrizes nacionais para o saneamento básico. Brasília. 2007.

CAIRNCROSS, C., FEACHEM, R. Environmental health engineering in the tropics: an introductory text. 2nd edition. Chichester, UK. 1993.

ENSP. Uma criança morre a cada 15 segundos devido a problemas relacionados à falta de água potável. 2013. Disponível em: 〈http://www6.ensp.fiocruz.br/visa/?q=node/4788 >. Acessado em 05 jul 2016.

FUNASA. Impactos na Saúde e no Sistema Único de Saúde Decorrentes de Agravos Relacionados a um Saneamento Ambiental Inadequado. FUNASA, Brasília, 2010. 246 p.

HELLER, L; CASSEB, M. L. M. Abastecimento de água. In: BARROS, R. T. V. et al (ORG.). Manual de Saneamento e Proteção Ambiental para os Municípios. Belo Horizonte. DESA/UFMG v. 2, 2001. 221 p.

HELLER, L. Saneamento e saúde. Brasília: OPAS/0MS, p.1-102, jun 1997.

IBGE. Projeção da população do Brasil. Rio de Janeiro: IBGE, 2008. Disponível em: $\langle$ http://seriesestatisticas.ibge.gov.br/series.aspx?no=18op=18vcodigo=P0P300\&t=revisa0-2008-projecaopopulacao-brasil $>$. Acessado em 26 maio 2016.

IBGE. Censo demográfico. Rio de Janeiro: IBGE, 2010. Disponível em: 〈http://www.ibge.gov.br/estadosat/l $>$. Acessado em 26 maio 2016.

IBGE. Populações rurais e urbanas no Censo Demográfico. Rio de Janeiro: IBGE, 2010. Disponível em:< http://www.censo2010.ibge.gov.br/sinopse/index.php?dados=8 >. Acessado em 06 de set 2016.

DATASUS. Ministério da Saúde. Doenças e Agravos de Notificação. Disponível em:

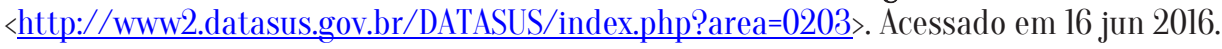


Avaliação preliminar dos efeitos da ineficiência dos serviços de saneamento na saúde pública brasileira

PLANSAB. Plano Nacional de Saneamento Básico. Ministério das Cidades. 2013.

RIPSA (a). Rede Interagencial de Informações para a Saúde. Gasto federal com saneamento como proporção do gasto federal total, 2012.

Disponivel em: 〈http://tabnet.datasus.gov.br/cgi/idb2012/el4.htm». Acessado em 14 maio 2016.

RIPSA (b). Rede Interagencial de Informações para a Saúde. Gasto federal com saúde como proporção do gasto federal total, 2012.

Disponível em: 〈http://tabnet.datasus.gov.br/cgi/idb2012/e08.htm〉. Acessado em 14 maio 2016.

SNIS. Sistema Nacional de Informações sobre Saneamento. Diagnóstico dos Serviços de Água e Esgoto - 2014. Brasília: SNSA/MCIDADES. 212 p. 2016 